\title{
Modelling the Effect of SPION Size in a Stent Assisted Magnetic Drug Targeting System with Interparticle Interactions
}

\author{
Adil Mardinoglu' ${ }^{1}$ and P. J. Cregg ${ }^{2}$ \\ ${ }^{1}$ Department of Biology and Biological Engineering, Chalmers University of Technology, 41296 Gothenburg, Sweden \\ ${ }^{2}$ Materials Characterisation \& Processing Group, Waterford Institute of Technology, Waterford, Ireland \\ Correspondence should be addressed to Adil Mardinoglu; adilm@chalmers.se
}

Received 28 September 2014; Accepted 1 February 2015

Academic Editor: Pradip Paik

Copyright (c) 2015 A. Mardinoglu and P. J. Cregg. This is an open access article distributed under the Creative Commons Attribution License, which permits unrestricted use, distribution, and reproduction in any medium, provided the original work is properly cited.

\begin{abstract}
Cancer is a leading cause of death worldwide and it is caused by the interaction of genomic, environmental, and lifestyle factors. Although chemotherapy is one way of treating cancers, it also damages healthy cells and may cause severe side effects. Therefore, it is beneficial in drug delivery in the human body to increase the proportion of the drugs at the target site while limiting its exposure at the rest of body through Magnetic Drug Targeting (MDT). Superparamagnetic iron oxide nanoparticles (SPIONs) are derived from polyol methods and coated with oleic acid and can be used as magnetic drug carrier particles (MDCPs) in an MDT system. Here, we develop a mathematical model for studying the interactions between the MDCPs enriched with three different diameters of SPIONs $(6.6,11.6$, and $17.8 \mathrm{~nm})$ in the MDT system with an implanted magnetizable stent using different magnetic field strengths and blood velocities. Our computational analysis allows for the optimal design of the SPIONs enriched MDCPs to be used in clinical applications.
\end{abstract}

\section{Introduction}

Cancer is a leading cause of death worldwide. Its cause is multifactorial and is linked to the interaction of genomic, environmental, and lifestyle factors [1]. Cancer patients are often diagnosed with localized reduction or loss of cellular control and normal maturation mechanisms that incorporate excessive cell growth, loss of cell differentiation, and the ability of cancerous tissue to grow into neighbouring tissues [2, 3]. Chemotherapy is one type of cancer treatment that inhibits the growth of, or kills, tumours. However, chemotherapy can damage healthy cells in the human body and it has many undesirable side effects [4]. It is therefore beneficial to alter the distribution of drugs in the human body, increasing the proportion of drugs at the target site while limiting concentration and effects in the rest of body through the use of Magnetic Drug Targeting (MDT) [5, 6]. Similar techniques have also been used to deliver other agents including cells [7].

MDT refers to the attachment of therapeutics to magnetizable particles to concentrate them at the desired locations by applying magnetic fields [8]. It includes the investigation of an external magnetic field and its interaction with biocompatible magnetic drug carrier particles (MDCPs) [9]. Significant difficulties in MDT are the inherently weak magnetic force relative to the hydrodynamic forces and targeting zones deep below the skin $[10,11]$. This makes MDCP collection problematic, because the magnetic force on a MDCP is proportional not only to the magnitude of the magnetic field but also to its gradient. To overcome these limitations, soft ferromagnetic materials such as wires, seeds, and stents are implanted into the body to increase the localized magnetic field strength and gradient, and this technique is called Implant Assisted Magnetic Drug Targeting (IA-MDT) [1215]. Different theoretical and clinical applications of IA-MDT have been developed [16-23]. Moreover, an IA-MDT system which uses a magnetizable stent as an implant and high gradient magnetic separation (HGMS) in a physiologically stretched vessel was studied with a $2 \mathrm{D}$ mathematical model [24]. In this Stent Assisted Magnetic Drug Targeting (SAMDT) system, a ferromagnetic stent was implanted to aid 
TABLE 1: Saturation magnetization $\left(M_{s}\right)$ of the oleate-capped $\mathrm{Fe}_{3} \mathrm{O}_{4}$ nanoparticles with different diameters at $300 \mathrm{~K}$.

\begin{tabular}{lc}
\hline Diameter $(\mathrm{nm})$ & Saturation magnetization $(\mathrm{kA} / \mathrm{m})$ \\
\hline 6.6 & 15.7 \\
11.6 & 14.7 \\
17.8 & 3.4 \\
\hline
\end{tabular}

collection of MDCPs in an elastic tube that has similar mechanical properties to the blood vessel and the changes in the mechanical behaviour were analyzed under the influence of mechanical forces generated.

There has been a growing interest in the scientific and clinical application of MDCPs as MDT vehicles for the development of efficient treatment strategies. A nanoparticlebased cancer drug has been developed and the phase 1 clinical study of cancer patients providing positive clinical evidence for the progress of nanoparticle application is reported [25]. Superparamagnetic iron oxide nanoparticles (SPIONs) have also been used as magnetic resonance imaging (MRI) contrast agents for labelling mammalian cells since their features can be easily tailored to include targeting moieties, fluorescence dyes, or therapeutic agents [26]. SPIONs can also be taken up by the cells through endocytosis and one particular SPION that contains ferumoxides is approved for hepatic imaging by the US Food and Drug Administration (FDA) [27].

The fraction of the constituting atoms on the surface of the nanoparticles varies with the decrease in the size of the particles $(<100 \mathrm{~nm})$ and this leads to significant changes in the magnetic structure and properties of the nanophase materials. The variation of the magnetic behaviour of welldispersed monodisperse $\mathrm{Fe}_{3} \mathrm{O}_{4}$ nanoparticles with respect to particle diameters $(6.6,11.6$, and $17.8 \mathrm{~nm})$ has been previously investigated [28]. It has been reported that the largest particles are ferromagnetic at room temperature and smaller nanoparticles exhibit superparamagnetism with the blocking temperatures increasing with the particle size. Saturation magnetization of the oleate-capped $\mathrm{Fe}_{3} \mathrm{O}_{4}$ nanoparticles with different diameters at $300 \mathrm{~K}$ is shown in Table 1 .

Here, we propose SPIONs as carriers in SA-MDT system using a magnetizable stent as an implant and focus on the theoretical modelling of the interaction between the MDCPs enriched with three different sizes of SPIONs (see Figure 1). The quantity of the SPIONs included in the MDCPs is inversely proportional to the diameter in SPIONs in each simulation. The quantity of the carrier particles at the desired site under the influence of four different magnetic field strengths and four different blood velocities is considered.

\section{Defining the SA-MDT System}

In order to optimize the size of the SPIONs in the content of MDCPs in an SA-MDT system, a 2D mathematical model was developed based on a previous model [18]. The mathematical model geometry of the SA-MDT system comprises a magnetizable coiled wire stent implant in close contact with the inner wall of a biological vessel. We expand the previous model [18] and present the next experimental challenge to develop a clinically relevant SA-MDT system. We model the behaviour of $N$ MDCPs under the influence of (i) Stokes drag, (ii) hydrodynamic interaction forces, and (iii) magnetic forces that account for the mutual magnetic dipole-dipole interactions and calculated (iv) the velocity of each MDCP and $\mathrm{MDCP}_{n}$ and (v) the system performance in terms of collection efficiency (CE) ignoring the effect of inertia and gravity (Figure 1).

The forces acting on a given particle labeled $n \mathrm{MDCP}_{n}$ are calculated as follows.

(i) Stokes drag, $\vec{F}_{S_{n}}$, decreases the blood velocity relative to the MDCPs and it can be written as

$$
\vec{F}_{s_{n}}=6 \pi \eta_{\text {blood }} R_{p_{n}}\left(\vec{v}_{\text {blood }}-\vec{v}_{p_{n}}\right) \text {, }
$$

where $\eta_{\text {blood }}$ is the viscosity of the blood, $R_{p_{n}}$ is the radius of $\mathrm{MDCP}_{n}$, and $\vec{v}_{\text {blood }}$ and $\vec{v}_{p_{n}}$ are the velocities of the blood and $\mathrm{MDCP}_{n}$, respectively. In the model, $\vec{v}_{\text {blood }}$ is determined by solving the appropriate Navier-Stokes equations as previously described [15].

(ii) Hydrodynamic interaction force, $\vec{F}_{\text {hyd }_{n}}$, is determined as the disturbance of the $\mathrm{MDCP}_{n}$ due to the movement of other MDCPs in the blood flow. By considering $N$ MDCPs, the force acting on $\mathrm{MDCP}_{n}$ due to the presence of the other $(N-1)$ MDCPs is given by

$$
\vec{F}_{\text {hyd }_{n}}=\sum_{\substack{i=1 \\ i \neq n}}^{N} \xi_{n i} \cdot\left(\vec{v}_{\text {blood }^{\prime}}-\vec{v}_{p_{i}}\right),
$$

where $\vec{v}_{p_{i}}$ is the velocity of $\mathrm{MDCP}_{i}$ and $\xi_{n i}$ is the modification due to the hydrodynamic interaction.

(iii) Magnetic forces acting on $\mathrm{MDCP}_{n}, \vec{F}_{m m_{n}}$, account for the externally applied magnetic field strength and mutual magnetic dipole-dipole interactions between MDCPs. Considering the $N$ MDCPs, each MDCP is taken as spherical and is having a homogeneous magnetic flux throughout the entire volume. $\vec{F}_{m m_{n}}$ on $\mathrm{MDCP}_{n}$ can be written as

$$
\vec{F}_{m m_{n}}=\left(\vec{m}_{n} \cdot \nabla\right) \vec{B}_{\text {Total } n},
$$

where $\vec{m}_{n}$ is the total magnetic moment of $\mathrm{MDCP}_{n}$ and $\vec{B}_{\text {Totaln }}$ is the total magnetic flux acting on $\mathrm{MDCP}_{n}$.

(iv) The velocity of $\mathrm{MDCP}_{n}, \vec{v}_{p_{n}}$, is obtained by summing the Stokes drag, the force due to hydrodynamic interaction, and the modified magnetic force (ignoring the inertial forces) as

$$
\begin{aligned}
& 6 \pi \eta_{\text {blood }} R_{p_{n}}\left(\vec{v}_{\text {blood }}-\vec{v}_{p_{n}}\right)+\sum_{\substack{i=1 \\
i \neq n}}^{N} \xi_{n i} \cdot\left(\vec{v}_{\text {blood }}-\vec{v}_{p_{i}}\right) \\
& +\left(\vec{m}_{n} \cdot \nabla\right) \vec{B}_{\text {Total } n}=0 .
\end{aligned}
$$




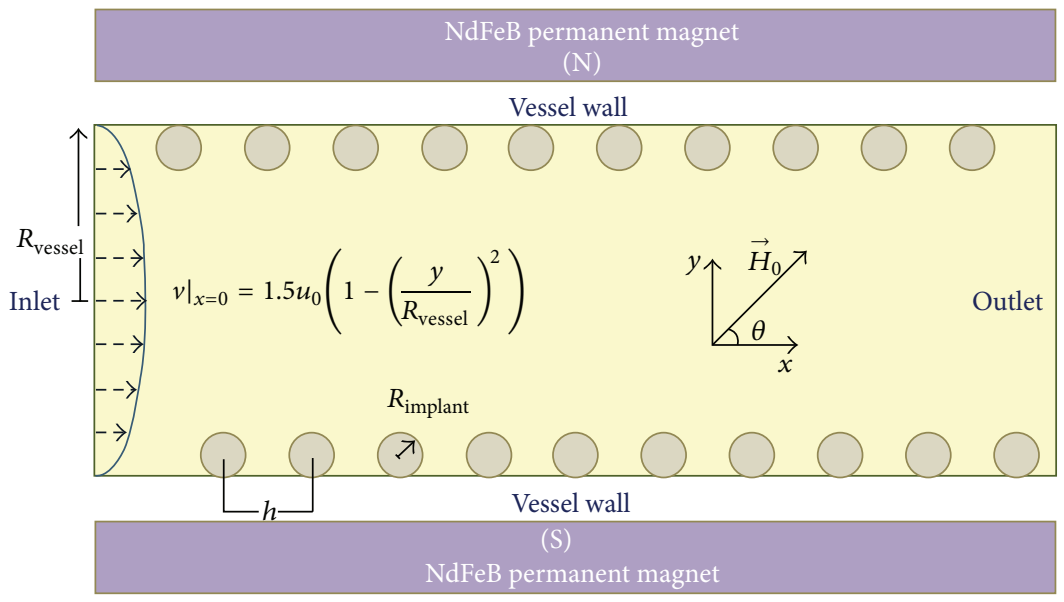

(a)

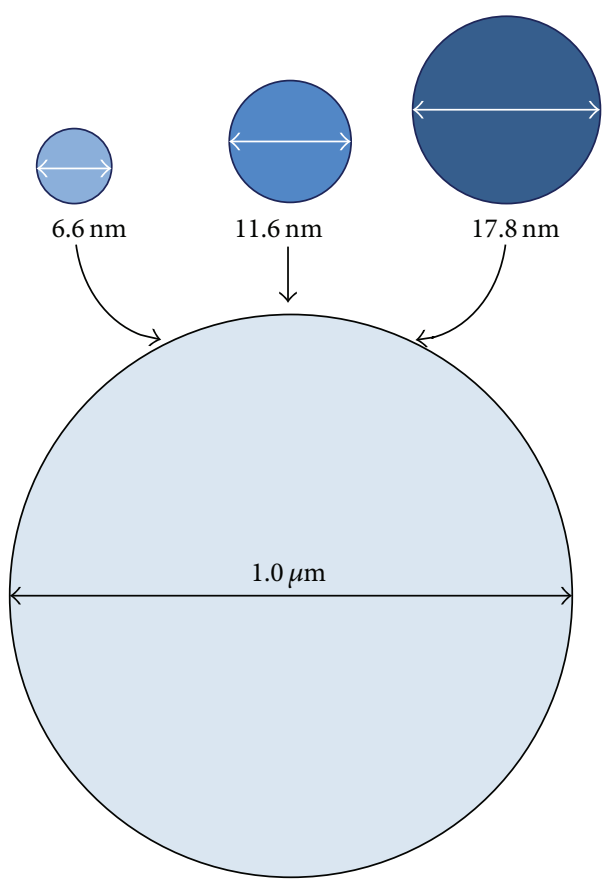

(b)

FIGURE 1: (a) Schematic of the CV used for studying the behaviour of SPIONs enriched MDCPs in SA-MDT system. (b) The modified magnetic force acting on MDCPs enriched with three different sizes of SPIONs is modelled in the SA-MDT system using different magnetic field strengths and blood velocities.

(v) The system performance of the model is calculated in terms of collection efficiency (CE) and the trajectories of $\mathrm{MDCP}_{n}$ are obtained from evaluating the streamline functions. Consider

$$
\mathrm{CE}=\frac{2 R_{\text {vessel }}-y_{1}+y_{2}}{2 R_{\text {vessel }}} 100 \% \text {, }
$$

where $R_{\text {vessel }}$ is the radius of the vessel and $y_{1}$ and $y_{2}$ are defined by the location of the streamline at the entrance of control volume (CV) of the last MDCPs captured by the stent wires.

\section{Results}

In the current study, we present the simulation results of the behaviour of MDCPs enriched with three different sizes of SPIONs (diameters 6.6, 11.6, and $17.8 \mathrm{~nm}$ ) in SA-MDT system. We examine the effects of interactions on the CE of the system in terms of the changes in blood velocity and applied magnetic field strength (Table 2).

We calculate the forces due to the magnetic dipole-dipole and hydrodynamic interactions on $N(N=100)$ MDCPs together with the blood flow velocity. Magnetic and hydrodynamic forces acting on MDCPs as well as blood velocity were calculated using the finite volume library OpenFOAM [29]. We create a homogeneously distributed square cloud of 100 MDCPs at the entrance of the CV, place the centre of the cloud at boundary of the reference capture cross section, simulate the behaviour of MDCPs at every time step considering their agglomeration, and eventually obtain the altered trajectories of MDCPs for calculating the CE. The number of the MDCPs is limited to 100 and the effective initial distance between the MDCPs at the entrance of CV is presented in Table 2 as calculated from a previous experimental setup [18]. In our previous studies, the number of MDCPs in the simulations has been limited to 25 leading to close agreement with the experimental results [18].

In order to describe the effect of different SPION diameter on the content of MDCPs, $\vec{F}_{m m_{n}}$ is calculated at the entrance of $\mathrm{CV}$ for each MDCP and presented in Figure 2. The saturation magnetization of SPIONs (oleate-capped, $\mathrm{Fe}_{3} \mathrm{O}_{4}$ nanoparticles) [28] is presented in Table 1. The number of the SPIONs in MDCP is inversely proportional to the diameter of the SPIONs. Improving the content and structure of the MDCPs and having better surface to volume ratio, we can have better applications of MDCPs in clinical studies.

Figure 2 shows the variation in CE of the SA-MDT system at four different applied magnetic field strengths $(0.25,0.50$, 0.75 , and $1 \mathrm{~T})$ and four different injection fluid velocities $(0.05$, $0.1,0.25$, and $0.5 \mathrm{~cm} / \mathrm{s}$ ). The resulting collection efficiencies derived from this mathematical model are in agreement with previously published work [18], and with differing SPION diameter, the system performance can differ by up to $20 \%$ in absolute terms.

\section{Discussions}

We have presented SA-MTD model incorporating the agglomeration of particles known to occur in real biological 
TABLE 2: Blood and material parameters used in the SA-MDT system.

\begin{tabular}{|c|c|c|c|}
\hline Properties & Symbol & Units & Values \\
\hline \multicolumn{4}{|l|}{ Applied field properties } \\
\hline Magnitude & $\mu_{0} H_{0}$ & $\mathrm{~T}$ & $0.25,0.50,0.75,1$ \\
\hline Angle of field direction & $\theta$ & - & $\pi / 2$ \\
\hline \multicolumn{4}{|l|}{ Physical properties } \\
\hline Temperature & $T$ & $\mathrm{~K}$ & 300 \\
\hline Boltzmann's constant & $k_{B}$ & $\mathrm{~J} / \mathrm{K}$ & $1.38 \times 10^{23}$ \\
\hline Permeability of vacuum & $\mu_{0}$ & $\operatorname{Tm} / \mathrm{A}$ & $4 \pi \times 10^{-7}$ \\
\hline \multicolumn{4}{|l|}{ MDCPs properties } \\
\hline Polymer material & - & - & $\mathrm{P}(\mathrm{S} / \mathrm{V}-\mathrm{COOH}) \mathrm{Mag}$ \\
\hline Radius & $R_{p}$ & $\mu \mathrm{m}$ & 0.5 \\
\hline MDCP concentration & - & Particle/L & $4 \times 10^{10}$ \\
\hline Density of the polymer material & $\rho_{\mathrm{pol}, p}$ & $\mathrm{~kg} / \mathrm{m}^{3}$ & 950 \\
\hline Initial distance between MDCPs & - & $\mu \mathrm{m}$ & 29.24 \\
\hline Saturation magnetization & $M_{p, s}$ & $\mathrm{kA} / \mathrm{m}$ & 22.4 \\
\hline \multicolumn{4}{|l|}{ Stent properties } \\
\hline Material & - & - & SS 430 \\
\hline Wire radius & $R_{\text {wire }}$ & $\mu \mathrm{m}$ & 62.5 \\
\hline Loop separation & $H$ & $\mathrm{~cm}$ & 0.2 \\
\hline Number of loops & $N_{l}$ & - & 10 \\
\hline Coil length & $L$ & $\mathrm{~cm}$ & 2 \\
\hline Saturation magnetization & $M_{\text {implant,s }}$ & $\mathrm{kA} / \mathrm{m}$ & 1261 \\
\hline Magnetic susceptibility & $X_{\text {implant }, 0}$ & - & 1000 \\
\hline \multicolumn{4}{|l|}{ Blood and vessel properties } \\
\hline Velocity & $u_{0}$ & $\mathrm{~cm} / \mathrm{s}$ & $0.05,0.1,0.25,0.5$ \\
\hline Volume & $V_{\text {blood }}$ & $\mathrm{mL}$ & 10 \\
\hline Density & $\rho_{b}$ & $\mathrm{~kg} / \mathrm{m}^{3}$ & 1000 \\
\hline Viscosity & $\eta_{b}$ & $\mathrm{~kg} / \mathrm{ms}$ & $1.0 \times 10^{-3}$ \\
\hline Vessel radius & $R_{\text {vessel }}$ & $\mathrm{cm}$ & 0.05 \\
\hline \multicolumn{4}{|l|}{ Magnetic material properties } \\
\hline Material & - & - & Oleate-capped $\mathrm{Fe}_{3} \mathrm{O}_{4}$ \\
\hline Weight content & $X_{\mathrm{fm}, p}$ & wt $\%$ & 100 \\
\hline Density & $\rho_{\mathrm{fm}, p}$ & $\mathrm{~kg} / \mathrm{m}^{3}$ & 5000 \\
\hline Magnetic moment & $m_{\mathrm{fm}, p}$ & $\mathrm{Am}^{2}$ & \\
\hline Saturation magnetization & $M_{\mathrm{fm}, p, s}$ & $\mathrm{kA} / \mathrm{m}$ & See Table 1 \\
\hline Diameter & $R_{\mathrm{fm}, p}$ & $\mathrm{~nm}$ & See Table 1 \\
\hline
\end{tabular}

systems and studied the effect of SPION diameter used in the MDCPs using different magnetic field strengths and blood velocities. We calculated both the dipole-dipole and hydrodynamic interactions for 100 particles and the resulting collection efficiencies derived from the mathematical model are in closer agreement with our latest experimental results [18].

We envisage that new insights obtained from the results of our analysis may be used in prediction of efficacy of targeted drug delivery for designing effective nanotherapeutic tools that can translate into the clinic. The CE of the system is increased with the higher magnetic field strength and decreased with the higher blood velocities as expected. Moreover, the modelling of different sizes of SPIONs in a SA-MDT system presented in this work represents a useful analytical tool for the prediction of the efficacy of targeted drug delivery. Our simulations indicate that size of the SPIONs in MDCPs together with saturation magnetization of the SPIONs has considerable effect on collection efficiency of the SA-MDT system. The response of SA-MDT is mainly dominated by the size of SPIONs and the saturation magnetization value of SPIONs, and these parameters can be calibrated based on the clinical applications of SA-MDT system using the results of our simulation. Improvement of the fundamental models in MDT systems may allow for the development of the more complex models that include systems level interactions.

The presented mathematical model for the movement of the MDCPs in the blood can be integrated with genomescale metabolic models (GEMs) for healthy cells/tissues [3033], cancers [34, 35], and cancer cell lines [36]. GEMs are 


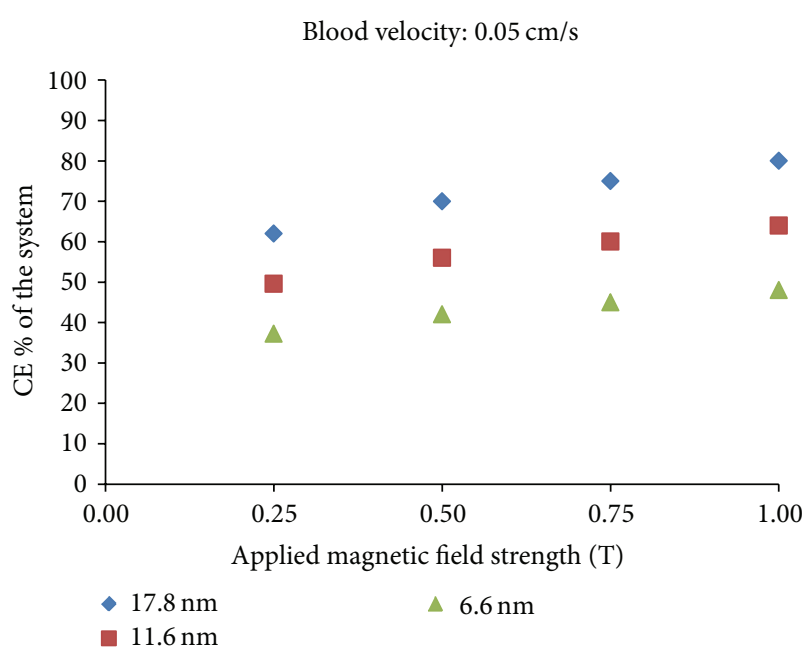

(a)

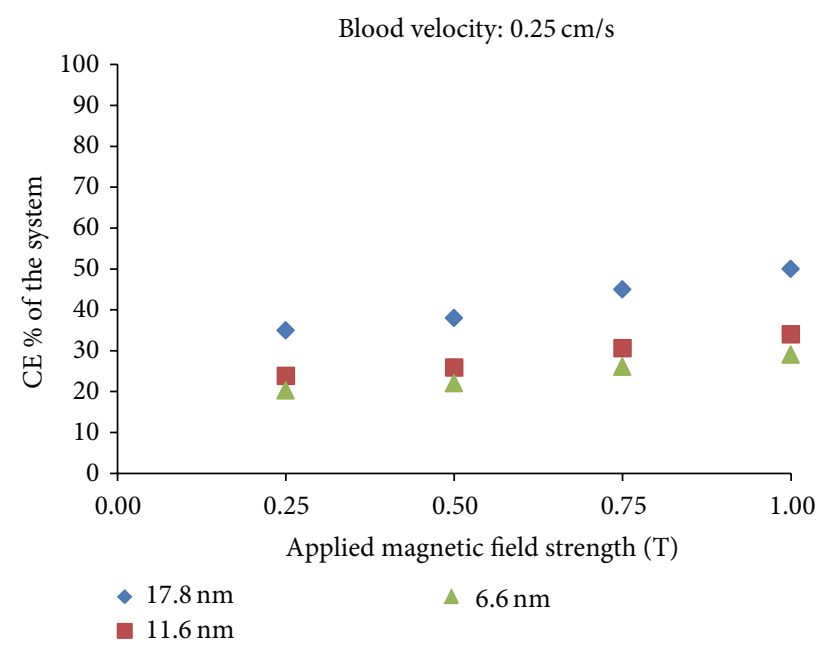

(c)

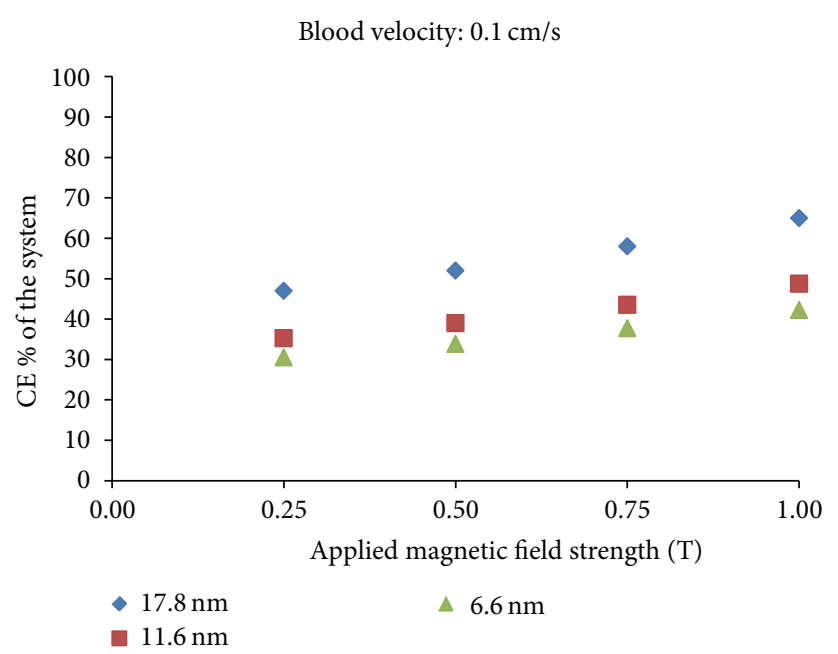

(b)

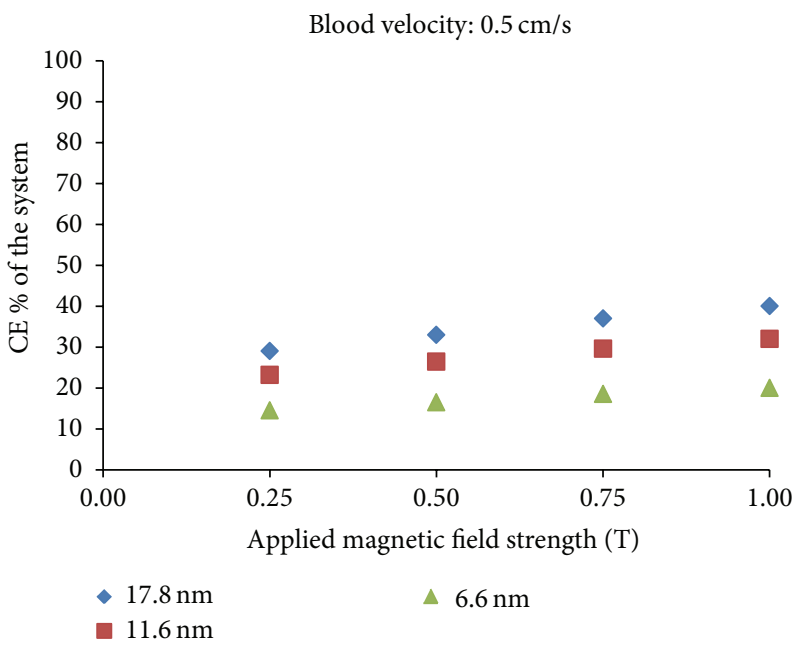

(d)

Figure 2: CE of the SA-MDT system is plotted with different magnetic field strengths $(0.25,0.50,0.75$, and $1 \mathrm{~T})$ and blood velocities $(0.05$, $0.1,0.25$, and $0.5 \mathrm{~cm} / \mathrm{s}$ ).

the compilation of biochemical reactions to define the entire known metabolism of the cells and tissues [37, 38], and they are reconstructed through the integration of proteomics [33, 39], transcriptomics [40-42], and metabolomics data [43]. Such integrative models can be used for discovery of novel biomarkers as well as for identification of drug targets to develop efficient treatment strategies for metabolism related diseases including cancer [44].

\section{Conflict of Interests}

The authors declare that they have no conflict of interests.

\section{Acknowledgments}

Dr. Adil Mardinoglu would like to thank the Knut and Alice Wallenberg Foundation and Dr. P. J. Creggwould like to thank Enterprise Ireland and the School of Engineering, WIT, for financial assistance to attend the 10th International Conference on the Clinical Applications of Magnetic Carriers, in Dresden, 2014. The authors would like to thank Dr. Adriele Prina-Mello for his comments and suggestions about the work.

\section{References}

[1] D. Hanahan and R. A. Weinberg, "Hallmarks of cancer: the next generation," Cell, vol. 144, no. 5, pp. 646-674, 2011.

[2] M. G. Vander Heiden, "Targeting cancer metabolism: a therapeutic window opens," Nature Reviews Drug Discovery, vol. 10, no. 9, pp. 671-684, 2011.

[3] D. A. Tennant, R. V. Durán, and E. Gottlieb, “Targeting metabolic transformation for cancer therapy," Nature Reviews Cancer, vol. 10, no. 4, pp. 267-277, 2010.

[4] S. H. Kaufmann and W. C. Earnshaw, "Induction of apoptosis by cancer chemotherapy," Experimental Cell Research, vol. 256, no. 1, pp. 42-49, 2000. 
[5] H. Kempe and M. Kempe, "The use of magnetite nanoparticles for implant-assisted magnetic drug targeting in thrombolytic therapy," Biomaterials, vol. 31, pp. 9499-9510, 2010.

[6] A. S. Lübbe, C. Alexiou, and C. Bergemann, "Clinical applications of magnetic drug targeting," Journal of Surgical Research, vol. 95, no. 2, pp. 200-206, 2001.

[7] J. Riegler, J. A. Wells, P. G. Kyrtatos, A. N. Price, Q. A. Pankhurst, and M. F. Lythgoe, "Targeted magnetic delivery and tracking of cells using a magnetic resonance imaging system," Biomaterials, vol. 31, no. 20, pp. 5366-5371, 2010.

[8] B. Shapiro, S. Kulkarni, A. Nacev, S. Muro, P. Y. Stepanov, and I. N. Weinberg, "Open challenges in magnetic drug targeting," Wiley Interdisciplinary Reviews: Nanomedicine and Nanobiotechnology, 2014.

[9] A. S. Lübbe, C. Bergemann, H. Riess et al., "Clinical experiences with magnetic drug targeting: a phase I study with 4'-epidoxorubicin in 14 patients with advanced solid tumors," Cancer Research, vol. 56, no. 20, pp. 4686-4693, 1996.

[10] G. Iacob, O. Rotariu, N. J. C. Strachan, and U. O. Häfeli, "Magnetizable needles and wires-modeling an efficient way to target magnetic microspheres in vivo," Biorheology, vol. 41, no. 5, pp. 599-612, 2004.

[11] A. Nacev, I. N. Weinberg, P. Y. Stepanov et al., "Dynamic inversion enables external magnets to concentrate ferromagnetic rods to a central target," Nano Letters, vol. 15, no. 1, pp. 359-364, 2015.

[12] B. B. Yellen, Z. G. Forbes, D. S. Halverson et al., "Targeted drug delivery to magnetic implants for therapeutic applications," Journal of Magnetism and Magnetic Materials, vol. 293, no. 1, pp. 647-654, 2005.

[13] A. J. Rosengart, M. D. Kaminski, H. T. Chen, P. L. Caviness, A. D. Ebner, and J. A. Ritter, "Magnetizable implants and functionalized magnetic carriers: a novel approach for noninvasive yet targeted drug delivery," Journal of Magnetism and Magnetic Materials, vol. 293, no. 1, pp. 633-638, 2005.

[14] J. A. Ritter, A. D. Ebner, K. D. Daniel, and K. L. Stewart, "Application of high gradient magnetic separation principles to magnetic drug targeting," Journal of Magnetism and Magnetic Materials, vol. 280, no. 2-3, pp. 184-201, 2004.

[15] A. Mardinoglu, Inclusion of interactions in mathematical modelling of implant assisted magnetic drug targeting [Ph.D. thesis], School of Engineering, Waterford Institute of Technology, Waterford, Ireland, 2009.

[16] P. J. Cregg, K. Murphy, and A. Mardinoglu, "Calculation of nanoparticle capture efficiency in magnetic drug targeting," Journal of Magnetism and Magnetic Materials, vol. 320, no. 23, pp. 3272-3275, 2008.

[17] P. J. Cregg, K. Murphy, and A. Mardinoglu, "Inclusion of magnetic dipole-dipole and hydrodynamic interactions in implantassisted magnetic drug targeting," Journal of Magnetism and Magnetic Materials, vol. 321, no. 23, pp. 3893-3898, 2009.

[18] P. J. Cregg, K. Murphy, A. Mardinoglu, and A. Prina-Mello, "Many particle magnetic dipole-dipole and hydrodynamic interactions in magnetizable stent assisted magnetic drug targeting," Journal of Magnetism and Magnetic Materials, vol. 322, no. 15, pp. 2087-2094, 2010.

[19] P. J. Cregg, K. Murphy, and A. Mardinoglu, "Inclusion of interactions in mathematical modelling of implant assisted magnetic drug targeting," Applied Mathematical Modelling, vol. 36, no. 1, pp. 1-34, 2012.

[20] H. Chen, A. D. Ebner, M. D. Kaminski, A. J. Rosengart, and J. A. Ritter, "Analysis of magnetic drug carrier particle capture by a magnetizable intravascular stent - 2: parametric study with multi-wire two-dimensional model," Journal of Magnetism and Magnetic Materials, vol. 293, no. 1, pp. 616-632, 2005.

[21] M. O. Avilés, A. D. Ebner, and J. A. Ritter, "Ferromagnetic seeding for the magnetic targeting of drugs and radiation in capillary beds," Journal of Magnetism and Magnetic Materials, vol. 310, no. 1, pp. 131-144, 2007.

[22] M. O. Avilés, A. D. Ebner, and J. A. Ritter, "Implant assistedmagnetic drug targeting: comparison of in vitro experiments with theory," Journal of Magnetism and Magnetic Materials, vol. 320, no. 21, pp. 2704-2713, 2008.

[23] J. O. Mangual, M. O. Avilés, A. D. Ebner, and J. A. Ritter, "In vitro study of magnetic nanoparticles as the implant for implant assisted magnetic drug targeting," Journal of Magnetism and Magnetic Materials, vol. 323, no. 14, pp. 1903-1908, 2011.

[24] A. Mardinoglu, P. J. Cregg, K. Murphy, M. Curtin, and A. PrinaMello, "Theoretical modelling of physiologically stretched vessel in magnetisable stent assisted magnetic drug targeting application," Journal of Magnetism and Magnetic Materials, vol. 323, no. 3-4, pp. 324-329, 2011.

[25] C. Sheridan, "Proof of concept for next-generation nanoparticle drugs in humans," Nature Biotechnology, vol. 30, no. 6, pp. 471473, 2012.

[26] J.-H. Lee, B. Schneider, E. K. Jordan, W. Liu, and J. A. Frank, "Synthesis of complexable fluorescent superparamagnetic iron oxide nanoparticles (FL SPIONs) and cell labeling for clinical application," Advanced Materials, vol. 20, no. 13, pp. 2512-2516, 2008.

[27] I. J. M. de Vries, W. J. Lesterhuis, J. O. Barentsz et al., "Magnetic resonance tracking of dendritic cells in melanoma patients for monitoring of cellular therapy," Nature Biotechnology, vol. 23, no. 11, pp. 1407-1413, 2005.

[28] D. Caruntu, G. Caruntu, and C. J. O’Connor, "Magnetic properties of variable-sized $\mathrm{Fe}_{3} \mathrm{O}_{4}$ nanoparticles synthesized from non-aqueous homogeneous solutions of polyols," Journal of Physics D: Applied Physics, vol. 40, no. 19, pp. 5801-5809, 2007.

[29] H. Jasak, "OpenFOAM: open source CFD in research and industry," International Journal of Naval Architecture and Ocean Engineering, vol. 1, no. 2, pp. 89-94, 2009.

[30] A. Mardinoglu, R. Agren, C. Kampf et al., "Integration of clinical data with a genome-scale metabolic model of the human Adipocyte," Molecular Systems Biology, vol. 9, article 649, 2013.

[31] A. Mardinoglu, R. Agren, C. Kampf, A. Asplund, M. Uhlen, and J. Nielsen, "Genome-scale metabolic modelling of hepatocytes reveals serine deficiency in patients with non-alcoholic fatty liver disease," Nature Communications, vol. 5, article 3083, 2014.

[32] A. Mardinoglu, C. Kampf, A. Asplund et al., "Defining the human adipose tissue proteome to reveal metabolic alterations in obesity," Journal of Proteome Research, vol. 13, no. 11, pp. 51065119, 2014.

[33] M. Uhlen, L. Fagerberg, B. M. Hallstrom et al., "Tissue-based map of the human proteome," Science, vol. 347, Article ID 1260419, 2015.

[34] R. Agren, S. Bordel, A. Mardinoglu, N. Pornputtapong, I. Nookaew, and J. Nielsen, "Reconstruction of genome-scale active metabolic networks for 69 human cell types and 16 cancer types using INIT,' PLoS Computational Biology, vol. 8, no. 5, Article ID e1002518, 2012.

[35] R. Agren, A. Mardinoglu, A. Asplund, C. Kampf, M. Uhlen, and J. Nielsen, "Identification of anticancer drugs for hepatocellular carcinoma through personalized genome-scale metabolic 
modeling," Molecular Systems Biology, vol. 10, no. 3, article A721, 2014.

[36] P. Ghaffari, A. Mardinoglu, A. Asplund et al., "Identifying antigrowth factors for human cancer cell lines through genomescale metabolic modeling," Scientific Reports, vol. 5, article 8183, 2015.

[37] A. Mardinoglu, F. Gatto, and J. Nielsen, "Genome-scale modeling of human metabolism-a systems biology approach," Biotechnology Journal, vol. 8, no. 9, pp. 985-996, 2013.

[38] A. Mardinoglu and J. Nielsen, "Systems medicine and metabolic modelling," Journal of Internal Medicine, vol. 271, no. 2, pp. $142-$ 154, 2012.

[39] M. Uhlen, P. Oksvold, L. Fagerberg et al., "Towards a knowledge-based Human Protein Atlas," Nature Biotechnology, vol. 28, no. 12, pp. 1248-1250, 2010.

[40] C. Kampf, A. Mardinoglu, L. Fagerberg et al., "The human liverspecific proteome defined by transcriptomics and antibodybased profiling," The FASEB Journal, vol. 28, no. 7, pp. 2901-2914, 2014.

[41] C. Kampf, A. Mardinoglu, L. Fagerberg et al., "Defining the human gallbladder proteome by transcriptomics and affinity proteomics," Proteomics, vol. 14, no. 21-22, pp. 2498-2507, 2014.

[42] L. Fagerberg, B. M. Hallstrom, P. Oksvold et al., "Analysis of the human tissue-specific expression by genome-wide integration of transcriptomics and antibody-based proteomics," Molecular and Cellular Proteomics, vol. 13, no. 2, pp. 397-406, 2014.

[43] D. S. Wishart, C. Knox, A. C. Guo et al., "HMDB: a knowledgebase for the human metabolome," Nucleic Acids Research, vol. 37, supplement 1, pp. D603-D610, 2009.

[44] A. Mardinoglu and J. Nielsen, "New paradigms for metabolic modeling of human cells," Current Opinion in Biotechnology, vol. 34, pp. 91-97, 2015. 

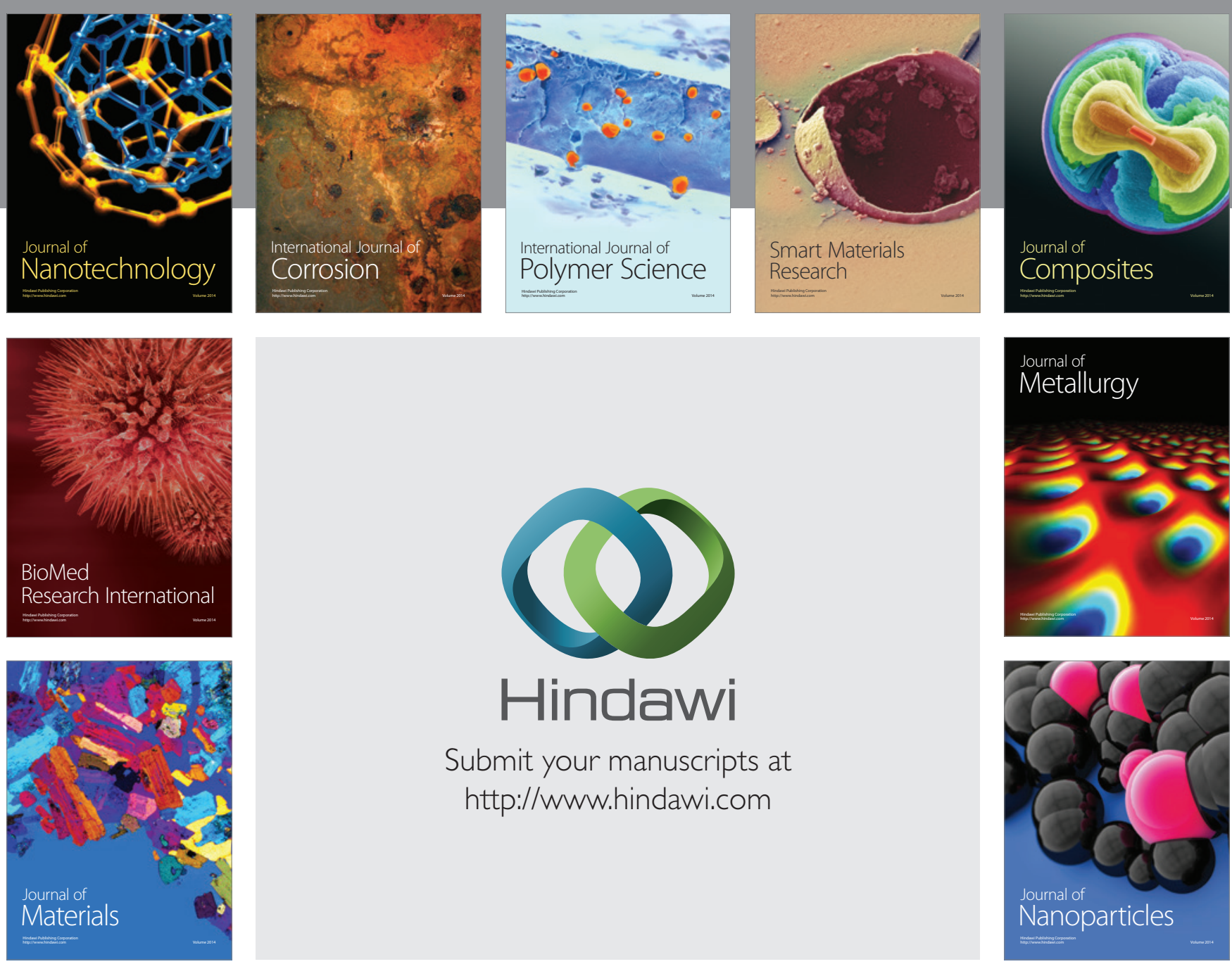

Submit your manuscripts at http://www.hindawi.com
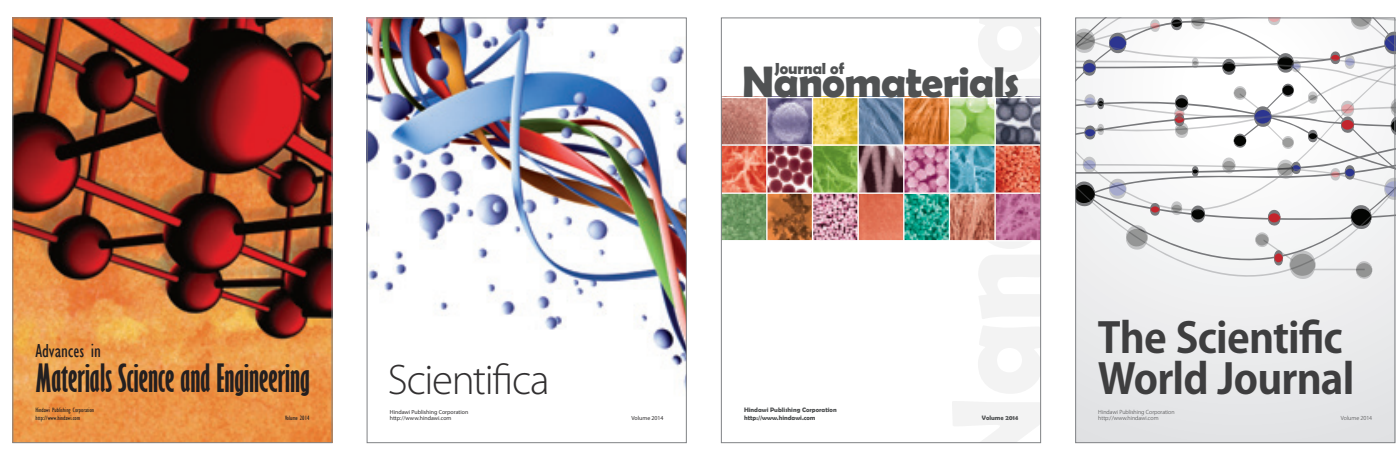

\section{The Scientific World Journal}
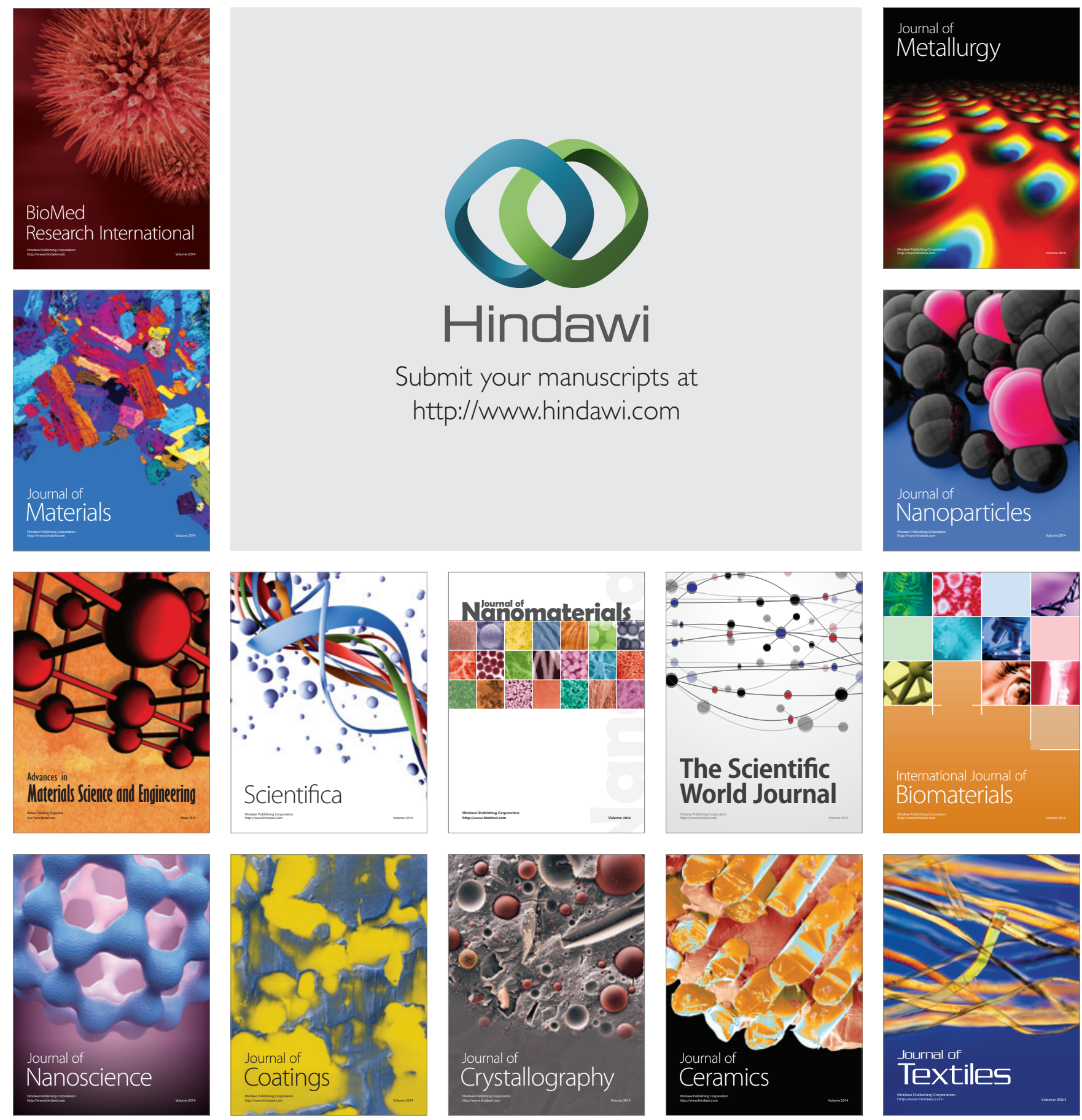